\title{
Bioinformatics Analysis of the Carotenoid Isomerase Gene in Cabbage (Brassica oleracea var. capitata)
}

\author{
Bo Sun, Min Jiang, Fen Zhang, and Haoru Tang ${ }^{\mathrm{a}, *}$ \\ College of Horticulture, Sichuan Agricultural University, Chengdu 611130, China \\ *,ahtang@sicau.edu.cn
}

\begin{abstract}
Carotenoid isomerase (CRTISO) is an important enzyme in carotenoid biosynthesis. Here, the Brassica oleracea var. capitata CRTISO (BocCRTISO) gene sequences were obtained from Brassica database (BRAD), and preformed for bioinformatics analysis. The BocCRTISO gene mapped to Scaffold000330, and contains an open reading frame of 1,773 bp that encodes a 590amino acid protein with a calculated molecular mass of $64.99 \mathrm{kD}$ and an isoelectric point (pl) of 6.75. Subcellular localization predicted the BocCRTISO gene was in the chloroplast. The conserved domain of the BocCRTISO protein is Rossmann-fold NAD $(P) H / N A D(P)(+)$ binding (NADB) domain. Homology analysis indicates that the CRTISO protein is apparently conserved during plant evolution and is most closely related to $\mathrm{B}$. napus, and $\mathrm{B}$. rapa. The findings of the present study provide a molecular basis for the elucidation of CRTISO gene function in cabbage.
\end{abstract}

Keywords: Carotenoid isomerase; gene sequences; bioinformatics.

\section{Introduction}

Cabbage (Brassica oleracea var. capitata) is a member of the Brassicaceae family that is widely distributed in the world. In China, cabbage is an important vegetable crop, and consumed considerable every years. Cabbage is generally grown for its leafy head as common edible part, which are crispy, tender, and tasty [1]. Besides its good flavor, cabbage is also a rich source of nutrients, antioxidants, and anticarcinogenic compounds, including carbohydrates, vitamin $\mathrm{C}$, glucosinolates, and carotenoids [1-2].

The enzymes involved in the carotenoid biosynthetic pathway have been extensively studied in various plants, including Arabidopsis [3], tomato [4], and citrus [5]. The first key step in carotenoid biosynthesis involves the production of a 40-carbon phytoene from two geranylgeranyl pyrophosphate (GGPP) molecules, which is catalyzed by phytoene synthase (PSY) [6-7]. Then, lycopene (colored carotenoid) is converted from phytoene (non-color carotenoid) by desaturases and isomerases, including phytoene desaturases (PDS) [8], $\zeta$-carotene desaturase (ZDS) [9], 15-cis- $\zeta$ carotene isomerase (Z-ISO) [10], and carotenoid isomerase (CRTISO) [3]. Hereafter, bifurcation of the carotenoid biosynthetic pathway occurs, and the production of $\beta$-carotene and $\alpha$-carotene is catalyzed by lycopene $\beta$-cyclase ( $\beta$-LCY) and lycopene $\varepsilon$-cyclase ( $\varepsilon$-LCY) [11-12].

CRTISO is an important enzyme in carotenoid biosynthesis, catalyzing the prolycopene into lycopene [13]. It is reported the identification of a CRTISO as the candidate gene for orange head by high-resolution genetic mapping using F2S4 population [14]. Loss of BrCRTISO function, upregulation of the upstream genes, and downregulation of downstream genes lead to the accumulation of prolycopene and confer an orange color to the inner head leaves in Chinese cabbage [15]. The genes encoding the CRTISO protein have been isolated in various plant species, including Arabidopsis [3], tomato [4], N. tabacum [16], and B. rapa [14]. To date, research studies on CRTISO in cabbage are limited. In the present study, the CRTISO gene sequence of cabbage was obtained from web database, and then bioinformatics analysis of the CRTISO gene were analyzed. The present study aimed to establish the foundation for further studies on the molecular mechanism of CRTISO in cabbage. 


\section{Materials and Methods}

\subsection{Sequence Obtain of the BocCRTISO Gene}

The genomic DNA and mRNA sequences of CRTISO gene of cabbage were downloaded and obtained from The Brassica database (BRAD) (http://brassicadb.org), and then used to subsequent bioinformatic analysis.

\subsection{Bioinformatics Analysis of the BocCRTISO Gene}

The amino acid sequence, protein molecular weight, isoelectric point, stability index, and hydrophobicity of the BocCRTISO gene were analyzed and predicted by ExPASy (http://web.expasy.org) and NCBI (https://www.ncbi.nlm.nih.gov/). Subcellular localization was predicted by WoLF PSORT (http://www.genscript.com/wolf-psort.html). The conserved domain were predicted by NCBI (https://www.ncbi.nlm.nih.gov/Structure/cdd/wrpsb.cgi). The amino acid sequence of CRTISO from eight additional species were downloaded from NCBI and subjected to multiple sequence alignment using DNAMAN. Phylogenetic tree analysis of the CRTISO proteins was executed in MEGA 6.0 using the neighbor-joining (NJ) method.

\section{Results}

\subsection{Analysis on Genomic Organization}

The genomic DNA and mRNA sequences of CRTISO gene of cabbage were downloaded and obtained from The Brassica database (BRAD) (http://brassicadb.org), and then used to subsequent bioinformatic analysis.

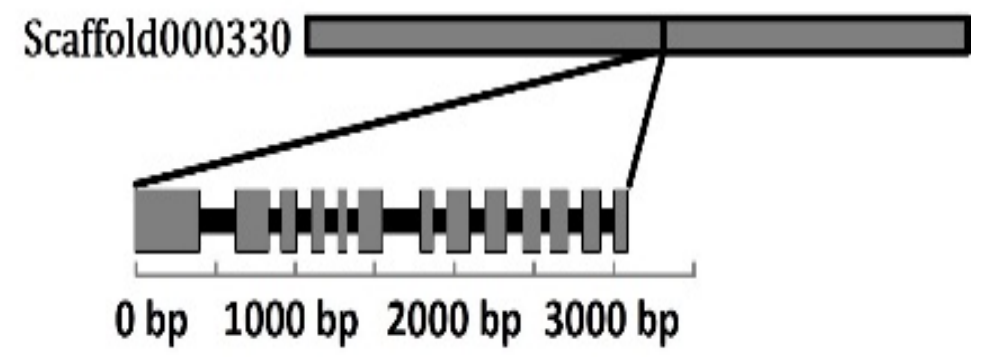

Exon $\boldsymbol{a}$ Intron

Figure 1. Chromosomal Location and Genomic Structure of BocCRTISO.

\subsection{Protein Physical and Chemical Properties Analysis}

Sequence analysis indicated that the BocCRTISO gene contained a 1,773-bp open reading frame (ORF), which encoded a 590-amino acids protein with a calculated molecular mass of $64.99 \mathrm{kD}$ and an isoelectric point $(\mathrm{pI})$ of 6.75. The amino acid types and proportions of the BocCRTISO gene was shown in Figure 2, the highest number of amino acid is Leucine (Leu), whereas the lowest number is Tryptophan (Trp). Its predicted formula was C2944H4612N768O851S19. Its total average hydrophilicity index was -0.079 , liposoluble index was 92.85 , and instability index in solution was 31.35 . 


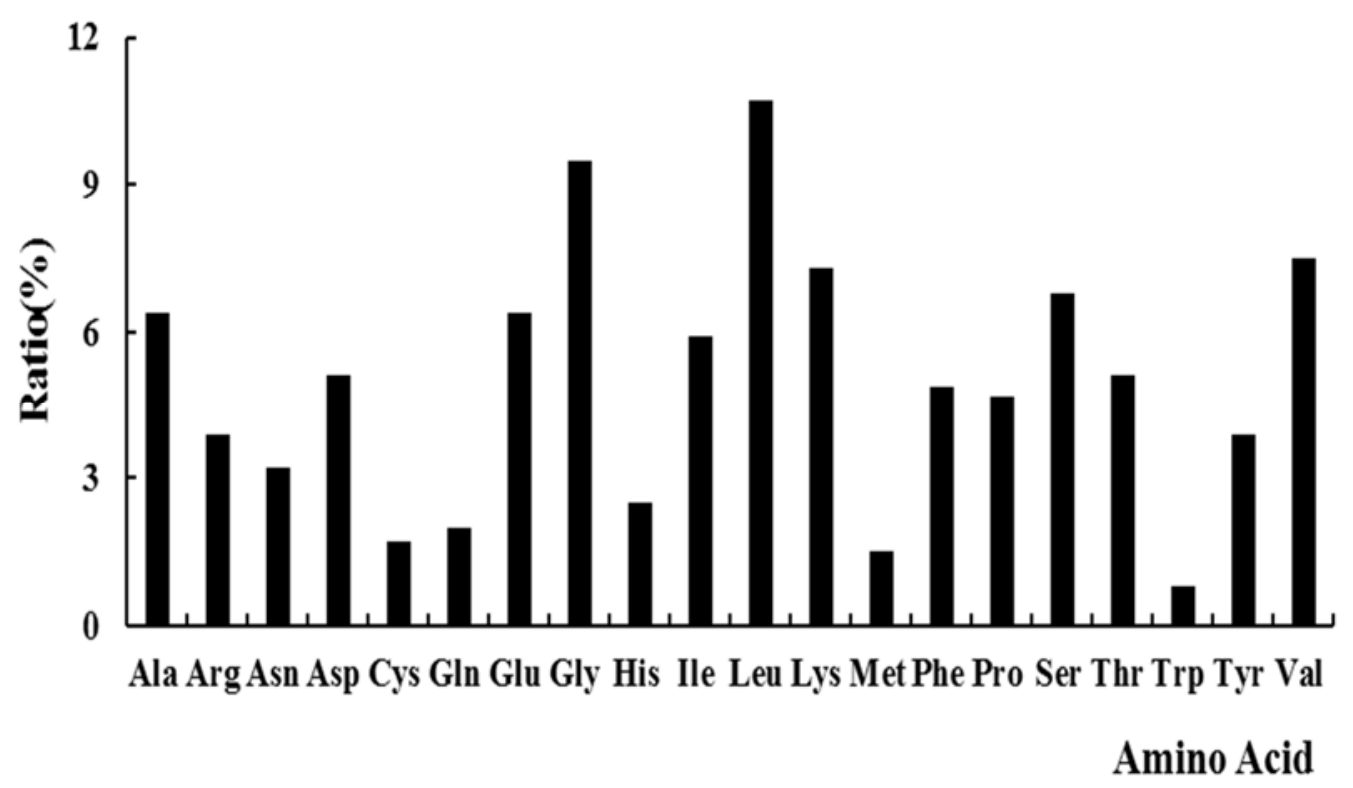

Figure 2. Amino acid Composition of BocCRTISO.

\subsection{Subcellular Localization and Conserved Domain Analysis}

Subcellular localization of the BocCRTISO gene was predicted by WoLF PSORT to be in the chloroplast. The analysis using Conserved Domain Database (CDD) demonstrated that the amino acid sequence of the BocCRTISO protein has one NADB Rossmann superfamily that share a Rossmann-fold NAD $(\mathrm{P}) \mathrm{H} / \mathrm{NAD}(\mathrm{P})(+)$ binding $(\mathrm{NADB})$ domain.

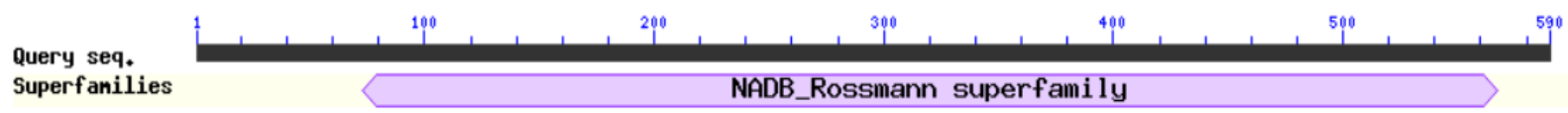

Figure 3. Conserved Domains Analysis of BocCRTISO.

\subsection{Homology and Phylogenetic Tree Analysis}

Homology analysis demonstrated that the amino acid sequence of the BocCRTISO protein shared high homology with those of other higher plant species. Figure 4 shows that the BocCRTISO had the highest identities with several CRTISO proteins of Cruciferae such as B. napus, B. rapa, Arabidopsis thaliana, and the levels of identity were $98 \%, 97 \%, 88 \%$ respectively. Interestingly, BocCRTISO showed $>76 \%$ identity with other species cited in our study, indicating that the CRTISO protein is highly conserved among different species. In addition, significant differences were found near the Ntermini of CRTISO proteins of various plant species (Fig. 4).

A phylogenetic tree was constructed to illustrate the relationship among the CRTISO proteins of cabbage and 19 other higher plant species (Fig. 5). A total of two major clusters were identified, one cluster includes Cruciferae, Euphorbiaceae, Sterculiaceae, and Moraceae, while the other cluster includes Solanaceae, Pedaliaceae, Rutaceae, and Rosaceae. Sequence alignment indicated that the BocCRTISO protein is more closely related to $\mathrm{B}$. napus and $\mathrm{B}$. rapa, which belonged to the Brassica branch. 


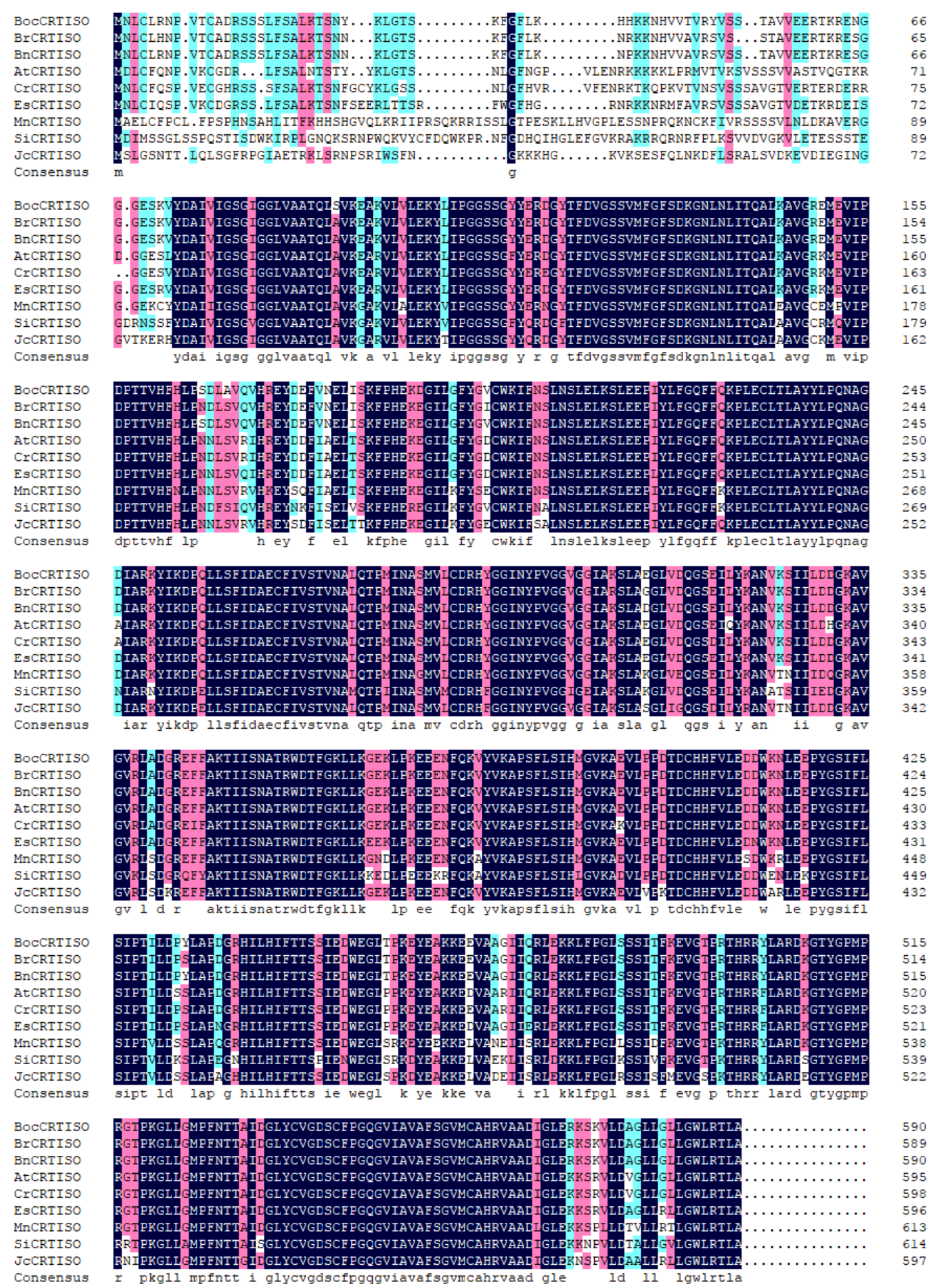

Figure 4. Amino Acid Sequence Alignment of BocCRTISO with the CRTISO Protein of other Species.

Boc: Brassica oleracea var. capitata; Br: Brassica rapa(XP 009118478.1); Bn: Brassica napus(CDY09954.1); At: Arabidopsis thaliana(NP_172167.2); Cr: Capsella rubella(XP_006307051.1); Es: Eutrema salsugineum(XP_006417877.1); Mn: Morus notabilis(XP_010110449.1); Si: Sesamum indicum(XP_011077785.1); Jc: Jatropha curcas(XP_012074260.1). 


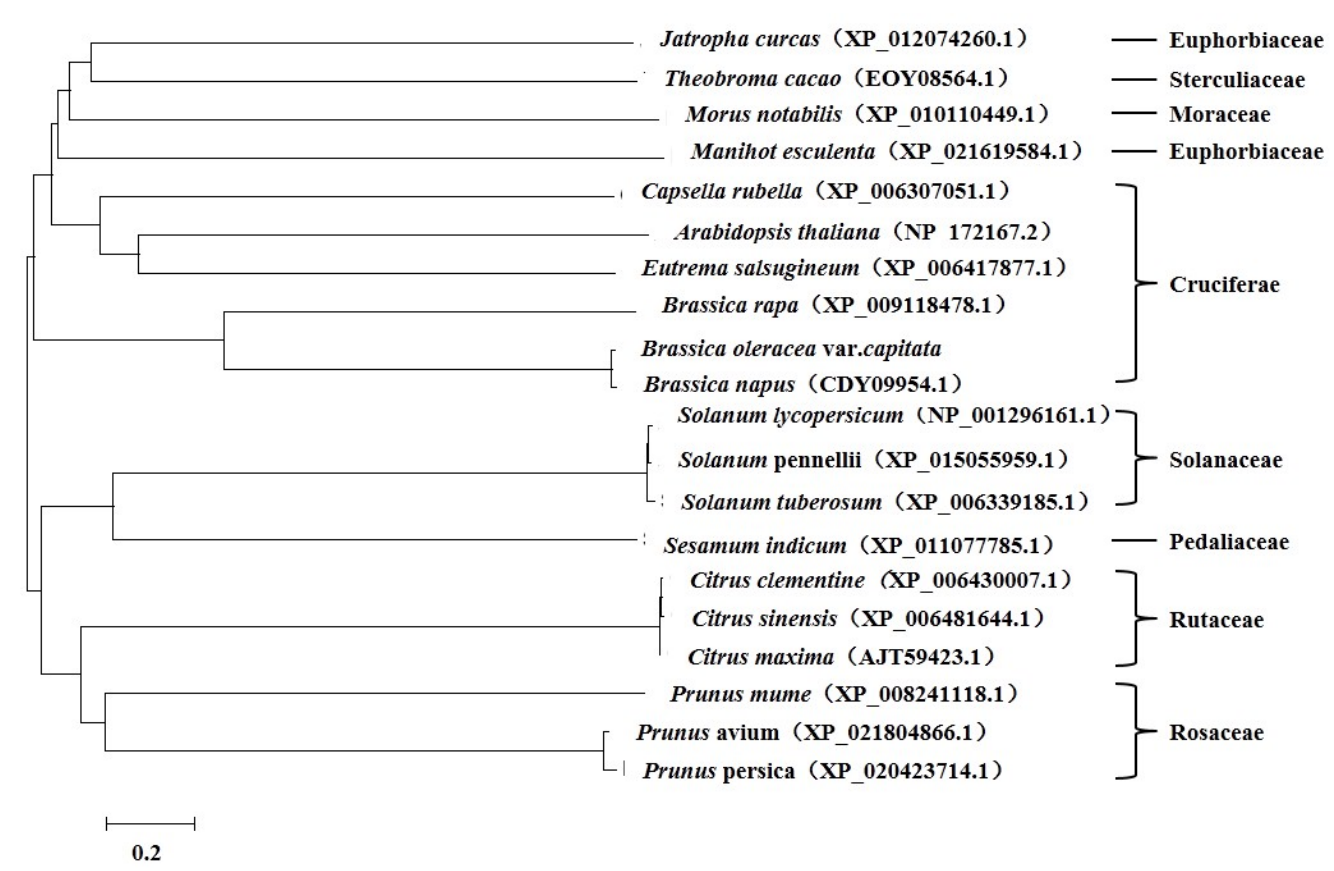

Figure 5. Phylogenetic Tree Analysis of BocCRTISO and CRTISO Proteins of other Species

\section{Discussion}

The present study analyzed the BocCRTISO gene of cabbage. CRTISO enzyme is encoded by single-copy genes in Arabidopsis [3], tomato [4], Chinese cabbage [14], and N. tabacum [16]. Similarly, the CRTISO gene occurred as a single copy in cabbage, indicating that the CRTISO enzyme may have undergone similar evolutionary patterns [17]. Previous studies have shown that the CRTISO protein is relatively conserved in plants [16]. The CRTISO protein of N. tabacum is similar to the CRTISO protein of tomato and potato, showing $93 \%$ and $93 \%$ homology [16]. The findings of the present study show that CRTISO from cabbage is highly conserved, particularly in plants; in the Cruciferae, these proteins show $>88 \%$ homology, similar to that observed in earlier reports. The findings of the present study may serve as a foundation for future studies on the functions of CRTISO in carotenoid metabolism in cabbage.

\section{Acknowledgments}

This work was supported by National Natural Science Foundation of China (31500247), key project of Department of Education of Sichuan Province (14ZA0016), and National Student Innovation Training Program (201710626030).

\section{References}

[1]. M. Wennberg, J. Ekvall, K. Olsson, and M. Nyman, Changes in carbohydrate and glucosinolate composition in white cabbage (Brassica oleracea var. capitata) during blanching and treatment with acetic acid, Food Chem. 95 (2006) 226-236.

[2]. S. Rokayya, C.J. Li, Y. Zhao, Y. Li, and C.H. Sun, Cabbage (Brassica oleracea L. var. capitata) phytochemicals with antioxidant and anti-inflammatory potential, Asian Pac. J. Cancer Prev. 14 (2014) 6657-6662.

[3]. H. Park, S.S. Kreunen, A.J. Cuttriss, D. Della Penna, and B.J. Pogson, Identification of carotenoid isomerase provides insight into carotenoid biosynthesis, prolamellar body formation, and photomorphogenesis, Plant Cell 14 (2002) 321-332. 
[4]. T. Isaacson, G. Ronen, D. Zamir, and J. Hirschberg, Cloning of tangerine from tomato reveals a carotenoid isomerase essential for the production of $b$-carotene and xanthophylls in plants, Plant Cell 14 (2002) 333-342.

[5]. M. Kato, Y. Ikoma, H. Matsumoto, M. Sugiura, H. Hyodo, and M. Yano, Accumulation of carotenoids and expression of carotenoid biosynthetic genes during maturation in critrus fruit, Plant Physiol. 134 (2004) 824-837.

[6]. P.A. Scolnik, and G.E. Bartley, Nucleotide sequence of an Arabidopsis cDNA for phytoene synthase, Plant Physiol. 104 (1994) 1471-1472.

[7]. P.R. Li, S.J. Zhang, S.F. Zhang, F. Li, H. Zhang, F. Cheng, J. Wu, X.W. Wang, and R.F. Sun, Carotenoid biosynthetic genes in Brassica rapa: comparative genomic analysis, phylogenetic analysis, and expression profiling, BMC Genomics 16 (2015) 492.

[8]. P.A. Scolnik, and G.E. Bartley, Phytoene desaturase from Arabidopsis, Plant Physiol. 103 (1993) 1475.

[9]. P.A. Scolnik, and G.E. Bartley, Nucleotide sequence of zeta-carotene desaturase (accession no. U38550) from Arabidopsis, Plant Physiol. 109 (1995) 1499.

[10]. Y. Chen, F. Li, and E.T. Wurtzel, Isolation and characterization of the Z-ISO gene encoding a missing component of carotenoid biosynthesis in plants, Plant Physiol. 153 (2010) 66-79.

[11]. B. Pogson, K.A. McDonald, M. Truong, G. Britton, and D. Della Penna, Arabidopsis carotenoid mutants demonstrate that lutein is not essential for photosynthesis in higher plants, Plant Cell 8 (1996) 1627-1639.

[12]. Y.M. Shi, R. Wang, Z.P. Luo, L.F. Jin, P.P. Liu, Q.S. Chen, Z.F. Li, F. Li, C. Y. Wei, M.Z. $\mathrm{Wu}$, P. Wei, H. Xie, L.B. Qu, F.C. Lin, and J. Yang, Molecular cloning and functional characterization of the lycopene $\varepsilon$-cyclases gene via virus-induced gene silencing and its expression pattern in Nicotiana tabacum, Int. J. Mol. Sci. 15 (2014) 14766-14785.

[13]. J.X. Zhang, H. Yuan, Z.J. Fei, B.J. Pogson, L.G. Zhang, L. Li, Molecular characterization and transcriptome analysis of orange head Chinese cabbage (Brassica rapa L. ssp. pekinensis), Planta 241 (2015) 1381-1394.

[14]. J.X. Zhang, H.X. Li, M.K. Zhang, M Hui, Q. Wang, L. Li, L.G. Zhang, Fine mapping and identification of candidate BR-or gene controlling orange head of Chinese cabbage (Brassica rapa L. ssp. pekinensis), Mol Breed 32 (2013) 799-805.

[15]. T.B. Su, S.C. Yu, J. Wang, F.L. Zhang, Y.J. Yu, D.S. Zhang, X.Y. Zhao, W.H. Wang, Loss of function of the carotenoid isomerase gene BrCRTISO confers orange color to the inner leaves of Chinese cabbage (Brassica rapa L. ssp. pekinensis), Plant Mol. Biol. Rep. 33 (2015) 648-659.

[16]. Y.M. Shi, R. Wang, J. Yang, Z.P. Luo, F. Li, M.Z. Wu, C.Y. Wei, F.C. Lin, L.B. Qu, P. Wei, Cloning and functional analysis of the CRTISO gene in Nicotiana tabacum, Acta Tabacaria Sinica 20 (2014) 138-143.

[17]. B. Sun, F. Zhang, X. Xia, S.L. Xue, Q. Yuan, Q. Chen, H.R. Tang, Cloning and expression analysis of BaPDS1 and BaPDS2 in Brassica alboglabra, Acta Horticulturae Sinica 43 (2016) 2257-2265. 